\title{
Performance Test of a Multi-cylindrical Magneto-rheological Fluid Clutch Demin Chen ${ }^{1, \text { a }}$, Qingge Cai ${ }^{2}$, Hong Zhang ${ }^{1, b}$ and Jinqiu Zhang ${ }^{1}$ \\ ${ }^{1}$ Academy of Armored Force Engineering, Beijing, China \\ ${ }^{2}$ Unit 63981 of PLA, China \\ admchn@163.com, bbitzhanghong@foxmail.com
}

Keywords: magneto-rheological fluid; multi-cylindrical; clutch; test study.

\begin{abstract}
To transmit a large torque and solve the effects of high speed rotating conditions on the properties of magneto-rheological fluid (MRF), a multi-cylindrical magneto-rheological fluid clutch (MRC) for B-class automobile is designed. The expressions of transmission torque are established. A sample MRC is manufactured. Use electronic torsion testing machine to test the torque transmission capability and stability, the combine and separate ability, and also the influence of remanence. The testing results indicate that the performance of MRC can achieve the goal.
\end{abstract}

\section{Introduction}

Magneto-rheological fluid clutch (MRC) is a kind of intelligent clutch which uses magneto-rheological effect to replace traditional friction to transmit torque. By controlling the currents in the coil to create different magnetic field, under which the magneto-rheological fluid (MRF) can form chain structure to transmit torque.

The present disc MRC only can transmit a small torque, mainly used as automobile fan clutch $[1,2,3,4]$. Moreover, the magnetic particles and the base fluid of the MRF in the disc MRC will be separated at high speed rotating conditions.

In this paper, a multi-cylindrical MRC for B-class automobile was designed to overcome those problems. Manufactured the clutch and made some tests to verify its performance.

\section{Structure of the MRC}

The transmission torque of the popular B-class automobile in the market is $375 \mathrm{~N} \cdot \mathrm{m}$. Set this as the target torque. The assembly drawing of the designed MRC is shown in figure 1.

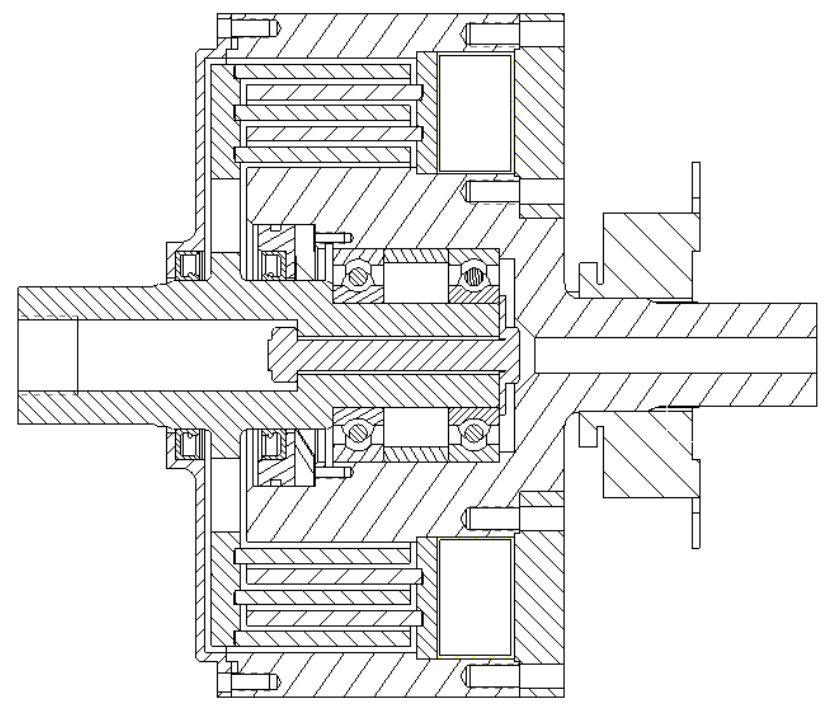

Fig.1 The assembly drawing of the MRC 
The MRC has three drive cylinders and two driven cylinders, formed 6 MRF work areas. The maximum size in radial is $230 \mathrm{~mm}$ and in axial is $124 \mathrm{~mm}$. This size can meet the space on the real automobile. Manufactured the MRC, the sample is shown in figure 2.

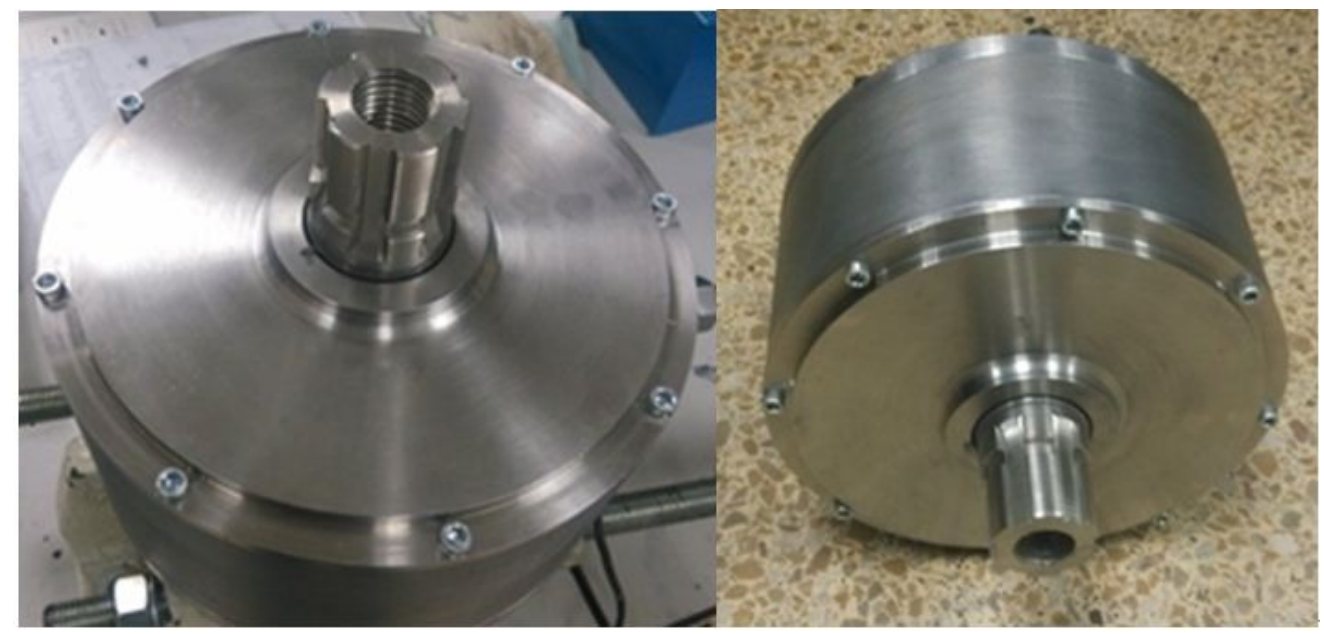

Fig. 2 The sample clutch

\section{Performance Test of the MRC}

The purpose of the test is to verify the MRC is able to transfer the target torque or not, and test the stability, the respond speed and the influence of the remanence during the torque transmission $[5,6,7]$. To simulate the power on the automobile, the biggest current used in the experiment is $3 \mathrm{~A}$.

The devices used in this experiment include ND-2000 type electronic torsion testing machine, power supply, data acquisition and control system. They are shown in Figure 3.

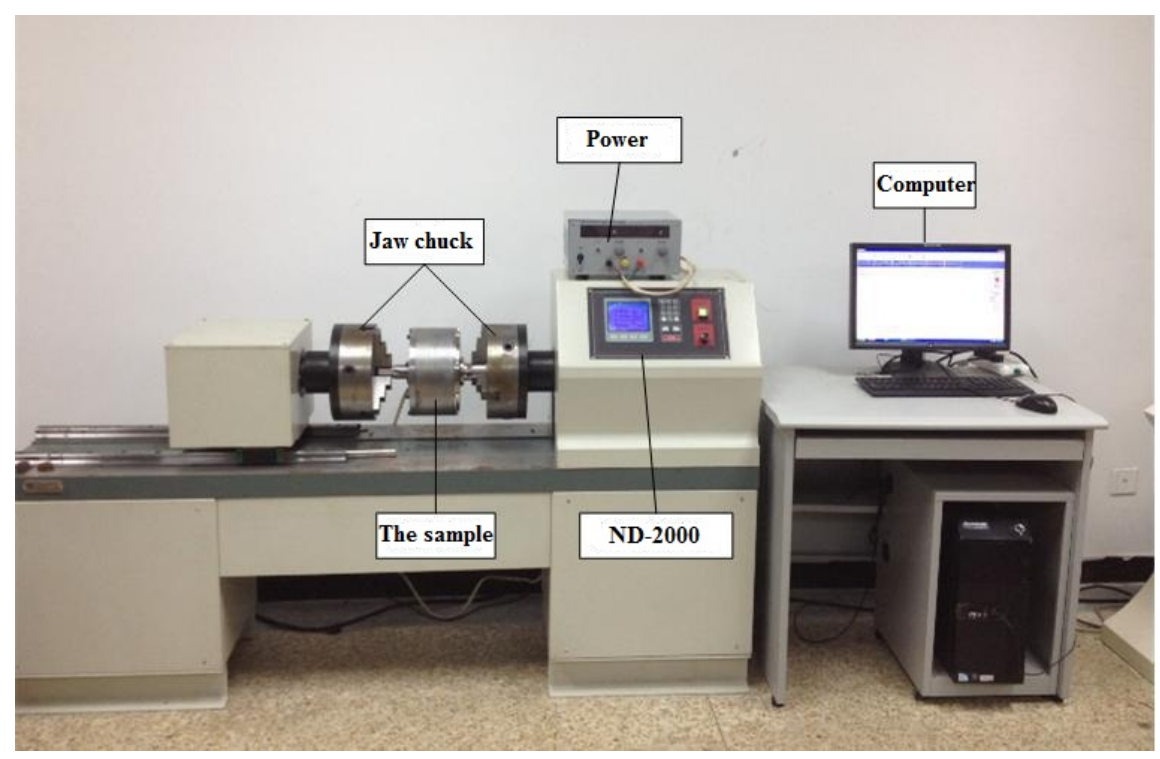

Fig.3 The devices used in this experiment

When there has no loading, the torque is created by the viscosity of the MRF and the friction of the bearings, as shown in figure 4.

From the results, the torque of non-loading under different current is less than $10 \mathrm{~N} \cdot \mathrm{m}$, the fluctuations is less than $3 \mathrm{~N} \cdot \mathrm{m}$. The effect can be neglected. 


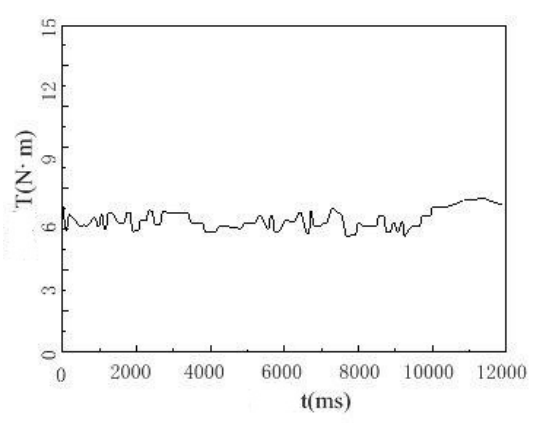

(a) $\mathrm{I}=0 \mathrm{~A}$

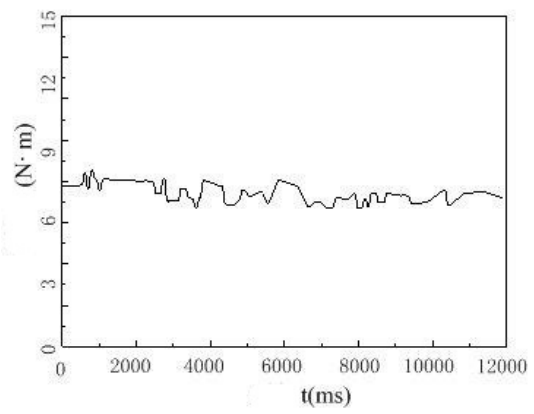

(c) $\mathrm{I}=1.0 \mathrm{~A}$

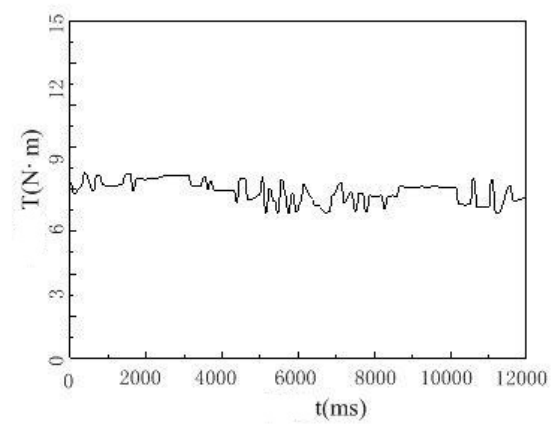

(e) $\mathrm{I}=2.0 \mathrm{~A}$

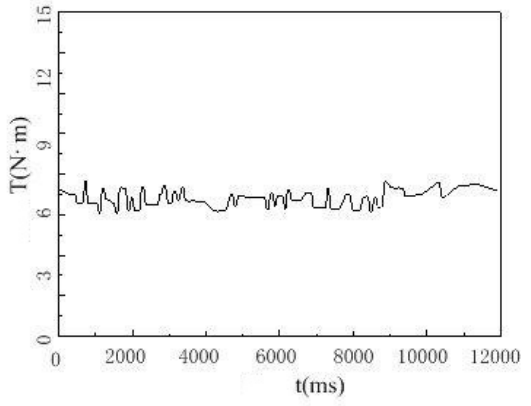

(b) $\mathrm{I}=0.5 \mathrm{~A}$

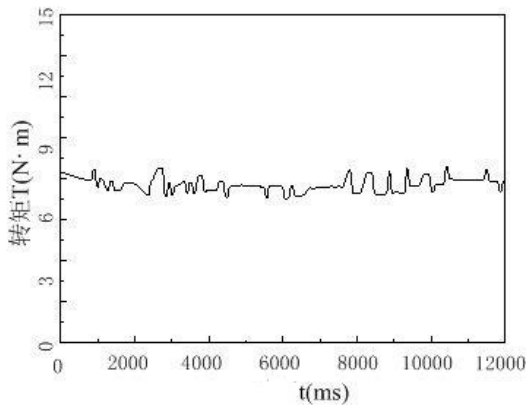

(d) $\mathrm{I}=1.5 \mathrm{~A}$

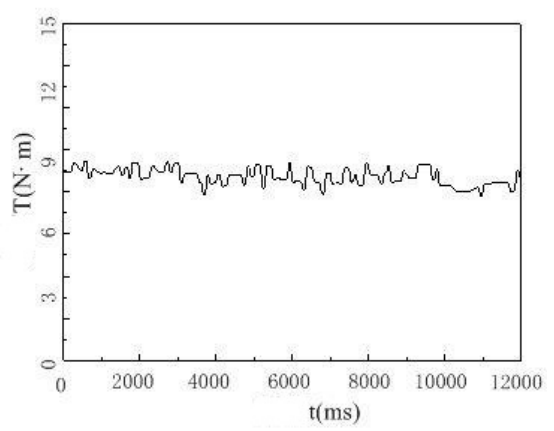

(f) $\mathrm{I}=2.5 \mathrm{~A}$

Fig.4 The Torque of non-loading

Torque Transmission Capability. Measure the transmission torque under the current of 0.5A, 1.0A, 1.5A, 2.0A, 2.5A, 3.0A respectively. Plot the data, got the relationship between the current and the torque, as shown in figure 5 . From the result, we got the transmission torque of a certain current, through which we can control the clutch. And the transmission torque under the current of $3 \mathrm{~A}$ is $410 \mathrm{~N} \cdot \mathrm{m}$, which achieved the target torque of $375 \mathrm{~N} \cdot \mathrm{m}$.

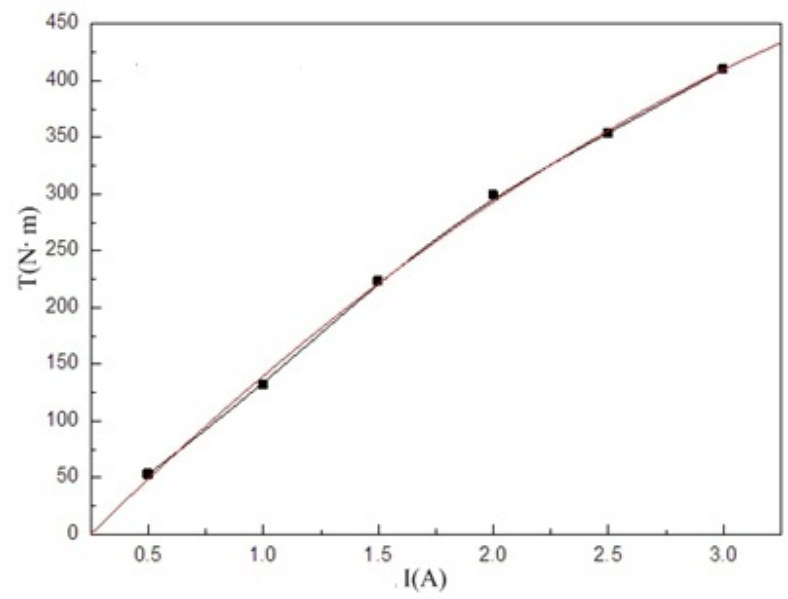

Fig.5 Torque-current curve

Torque Transmission Stability. Observe the transmission torque of a certain current which last for $12 \mathrm{~min}$. The result is shown in figure 6. From the result, the torque transmission is stable. 


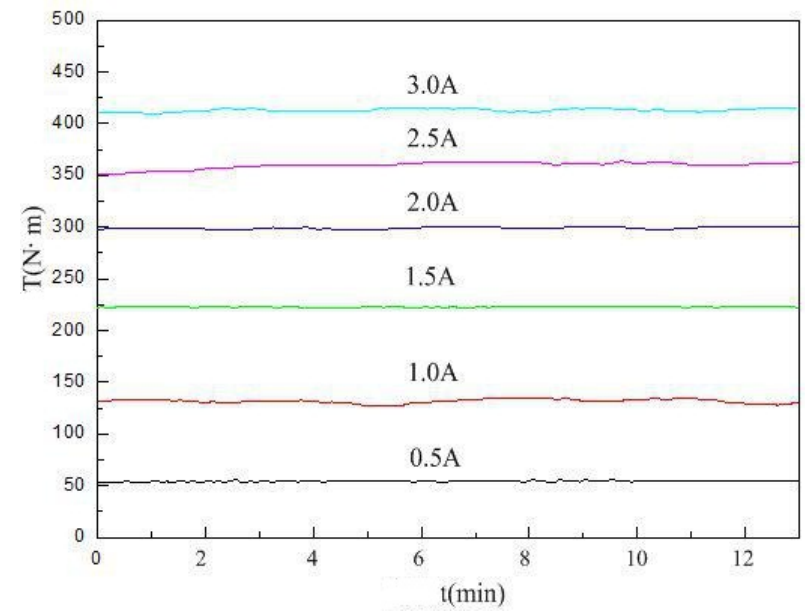

Fig.6 The torque transmission stability

Combine and Separate Ability. By loading and unloading the current in the coil continuously to test the combine and separate ability of the MRC, the result is shown in figure 7.

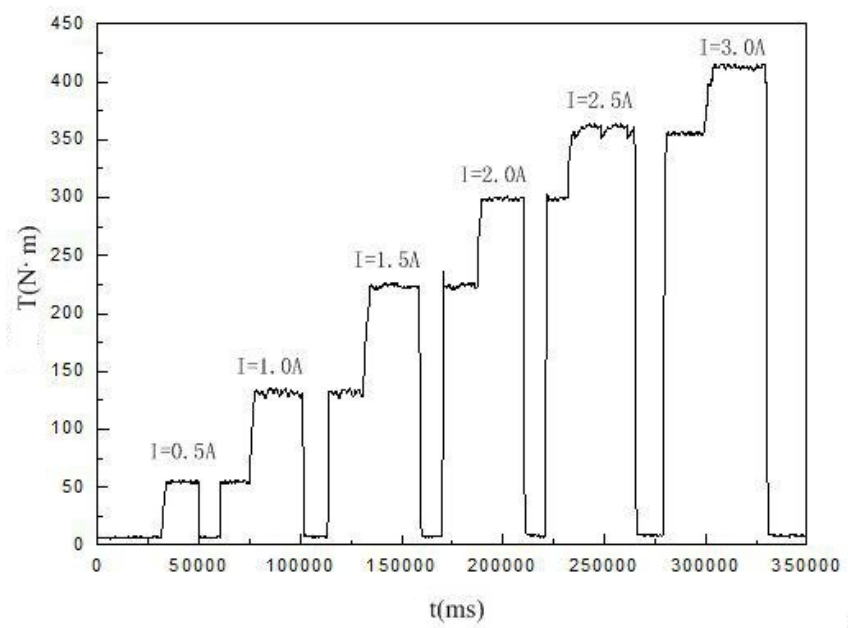

Fig.7 Combine and separate ability

While loading and unloading the current, the torque changes immediately. The combination and separation of the MRC is quickly. Measuring the combination and separation time, the results are shown in figure 8.

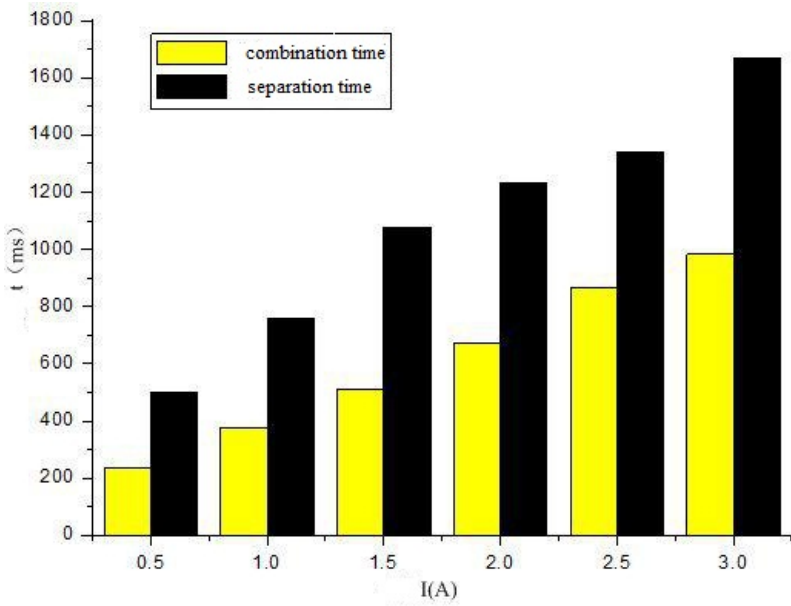

Fig.8 The combination and separation time

We can see from the results that the shortest combination time is 234ms and longest is 982ms; the shortest separation time is $500 \mathrm{~ms}$ and the longest is $1672 \mathrm{~ms}$. The combination and separation 
time is increasing with the increasing of the current. Under a same current, the separation time is longer than combination time.

Influence of Remanence. In order to find out the influence of remanence, we load the current from $0 \mathrm{~A}$ to $3 \mathrm{~A}$, and then unload. Measure the torque under the same current. The result is shown in figure 9 . From that, the torque of the unloading condition is bigger than that of loading at the same current, but the fluctuations is less than $25 \mathrm{~N} \cdot \mathrm{m}$.

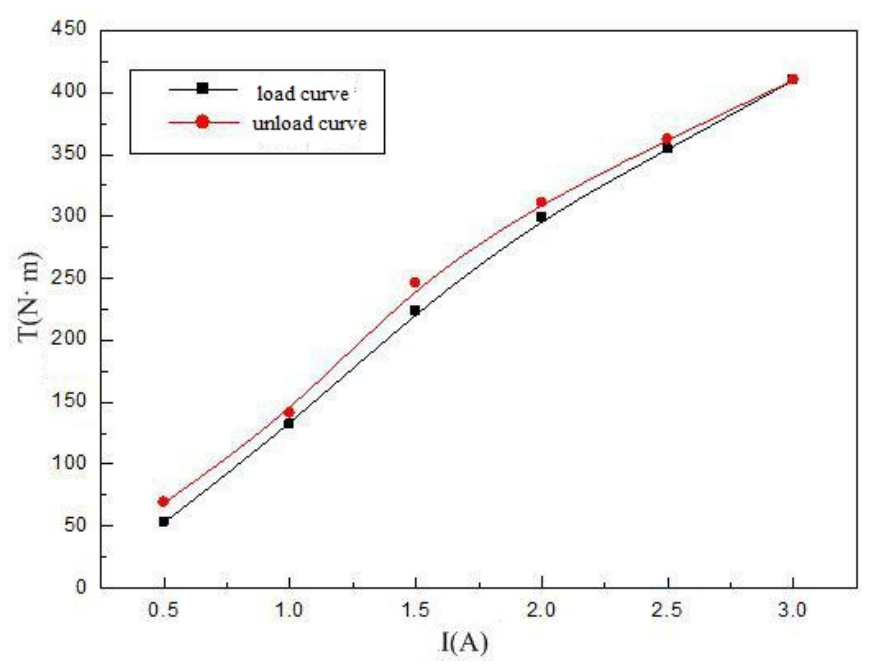

Fig.9 The influence of remanence

\section{Summary}

From the test results, the MRC has a good transmission capability and stability. The MRC can transmit a torque of $410 \mathrm{~N} \cdot \mathrm{m}$ when the current is $3 \mathrm{~A}$, which can fulfill the target torque of B-class vehicles. The combination and separation time of the MRC is quickly, and the remanence has a little influence to the MRC. 


\section{References}

[1] Chen D M, Cai Q G, Zhang H. Simulation and Test Study of Multidisc Magneto-Rheological Fluid Clutch[J]. Applied Mechanics \& Materials, 2014, 599-601.

[2] Pan C. The Design and Experimental Study of Magneto-rheological Fluid Fan Clutch[J]. Automotive Engineering, 2005.

[3] Shen Y, Pan C, Zhang J. Study on Fuzzy Intelligent Control System for Magneto-rheological Fluid Clutch of Automobile Fan[J]. Journal of Shijiazhuang Railway Institute, 2005.

[4] Horváth, P, T02r02csik, D. Optimization of A Disc-Type Magneto-Rheological Clutch[J]. Scientific Proceedings Faculty of Mechanical Engineering, 2012, 19(1):106-111.

[5] Shen Y, Yang S, Pan C. Experimental research of magneto-rheological fluid clutch[C]// Vehicular Electronics and Safety, 2005. IEEE International Conference on. IEEE, 2005:104 107.

[6] Pan C. The Design and Experimental Study of Magneto-rheological Fluid Fan Clutch[J]. Automotive Engineering, 2005.

[7] Shen Y, Yang S, Pan C, et al. Design and Experimental Research of Magneto-Rheological Fluid Fan Clutch[J]. Asme International Design Engineering Technical Conferences \& Computers \& Information in Engineering Conference, 2005. 